during the same period (226 women, 192 men), controlling for age, education, marital status, prior family bereavement, number of life events since M2, and health status. Biological health risks were assessed by allostatic load score, summing 7 subscales of physiological dysregulation (e.g., sympathetic, parasympathetic, inflammation, cardiovascular dysregulation). The results show that women who experienced bereavement reported poorer self-rated health longitudinally and had a higher level of allostatic load at biomarker data collection, which was significantly associated with poorer self-rated health approximately 10 years later. For men, however, bereavement was not associated with poorer self-rated health. In addition, the results indicate that coping moderated this relationship; bereaved women who reported higher levels of coping were less vulnerable to the detrimental longitudinal impacts of bereavement on health. Overall, the findings suggest that bereavement has longitudinal adverse health impacts via allostatic load for women, contingent on their coping strategies.

\section{CUMULATIVE EXPOSURE TO AIR POLLUTION AND SUBSEQUENT MORTALITY AMONG OLDER ADULTS} IN CHINA

J. Zhang ${ }^{1}$, S. McLaughlin ${ }^{2}$, L. Li ${ }^{3}$, 1. Fudan University, 2. Miami University, 3. University of Michigan

Research on the health effects of exposure to air pollution is growing. However, relatively little attention has been paid to the effects of long-term and cumulative exposure to air pollutants. Individual-level studies on the health consequences of air pollution in China are especially scarce. The purpose of this study is to examine the effect of cumulative exposure to sulfur dioxide (SO2), an air pollutant of particular concern in China, on all-cause mortality in older Chinese adults. Using a nationally representative sample of older adults in China $(\mathrm{N}=11,199)$, we tracked mortality over an 11-year period (2000-2011). Air pollution data were linked to respondents using provincial identifiers. To examine the effect of cumulative $\mathrm{SO} 2$ exposure on mortality, we employed multilevel multinomial logistic regression models that account for within subject clustering of observations over time as well as clustering at the province level. We found that every $10-\mu \mathrm{g} / \mathrm{m} 3$ increase in cumulative exposure to SO2 increased the odds of death by nearly $1 \%$ $(\mathrm{OR}=1.008 ; 95 \% \mathrm{CI}, 1.002-1.014)$, controlling for provinceand individual-level social and economic characteristics. Our analysis shows that air pollution is a risk factor for morality in older Chinese adults. Findings suggest that stronger $\mathrm{SO} 2$ regulations may enhance longevity.

\section{EFFECT OF LIFE SPAN EXPERIENCE WITH} ADVERSITIES AND TRAUMA ON SUBJECTIVE WELLBEING AMONG OLDER COMMUNITY DWELLERS

\section{Yang, D. Hedeker, University of Chicago}

Using the life course guidance, the goal of this study was to see if whether trauma experienced during various time points (childhood, middle-age and later life) changed across time for the subjects with respect to subjective well-being among older adults. Very little research has focused on how older community dwelling foreign born exposed to adversities during different points in the life course fare in relation to subjective well-being as defined by life satisfaction, depression, and self-rated health. This study employed data from the Health and Retirement study (1992-2012) $(\mathrm{N}=5,666)$ to examine these trends over time. We used multilevel models to test for specific individual change across time. The mean age for this group was $70.9(\mathrm{SD}=8.8)$. The majority of the respondents were female $(60 \%)$. About $6 \%$ were foreign born non-Hispanic Whites, $12 \%$ were nonHispanic Blacks, 2\% were non-Hispanic other, 9\% were Hispanic, and $78 \%$ were non-Hispanic Whites. In terms of early childhood adversities, $77 \%$ had experienced at least one trauma and $72 \%$ has experienced at least one trauma in adulthood. Findings suggested exposure to childhood adversities and adulthood trauma increases depression, poor self-rated health, and low satisfaction of life over time. Foreign born (non-Hispanic Whites) older adults suffered from depression, rated their health poor, and had lower life satisfaction compared to their counterparts. Findings from this study provide insight into how life course exposure of adversities and trauma among older adults showed a negative trend over time.

\section{GENOME-WIDE ASSOCIATION STUDY OF A HEALTHY METABOLIC PHENOTYPE IN THE LONG LIFE FAMILY STUDY}

M. Marron ${ }^{1}$, I. Miljkovic ${ }^{1}$, M. Wojczynski ${ }^{2}$,

R. Boudreau ${ }^{3}, K$. Christensen ${ }^{4}$, B. Thyagarajan 5 ,

J. Zmuda ${ }^{1}$, A. Newman ${ }^{1}$, 1. University of Pittsburgh, 2.

Washington University School of Medicine in St. Louis, 3.

Dept of Epidemiology, Univ of Pittsburgh, 4. University of

Southern Denmark, 5. University of Minnesota

Familial longevity has been shown to be associated with healthy metabolic characteristics. We sought to develop a healthy metabolic phenotype in the Long Life Family Study to identify potential genes regulating metabolic health. The Long Life Family Study is a multi-center cohort of twogeneration families selected for familial longevity. Using information from participants in the offspring generation, we developed a healthy metabolic phenotype by applying a Latent Profile Analysis to age- and gender-adjusted z-scores of body mass index, waist circumference, and fasting serum levels of glucose, insulin, triglycerides, high-density lipoprotein cholesterol, interleukin-6, and C-reactive protein. A subset of offspring-generation participants were defined as meeting the healthy metabolic phenotype if the Latent Profile Analysis classified them into a subgroup with a healthier profile of metabolic markers than expected given their age and gender, relative to the entire Long Life Family Study offspring generation. The log odds of meeting the healthy metabolic phenotype was used to calculate heritability and to apply a genome-wide association study. Among 1987 participants in the offspring generation $(43 \%$ men; mean (standard deviation) age and body mass index: $60(8)$ years and $28 \pm 5 \mathrm{~kg} /$ $\mathrm{m} 2), 388(20 \%)$ met the healthy metabolic phenotype. The $\log$ odds of meeting the healthy metabolic phenotype was heritable $(h 2=0.40, p<0.001)$, with suggestive associations $(\mathrm{p} \leq 8.7 \mathrm{E}-07)$ detected near genes: LOC101927936, PRSS23, ZPR1, LOC105372224, and LOC105377365 (on 11p15.4, 11q14.2, 11q23.3, 18q23, and 4q25, respectively). Further investigation may indicate biologic pathways influencing metabolic health and longevity in the Long Life Family Study cohort. 